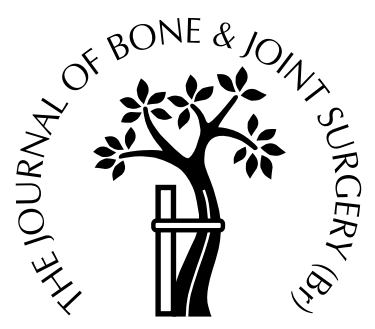

\title{
Mechanical factors in the initiation and propagation of tears of the rotator cuff
}

\section{QUANTIFICATION OF STRAINS OF THE SUPRASPINATUS TENDON IN VITRO}

\author{
P. Reilly, A. A. Amis, A. L. Wallace, R. J. H. Emery \\ From Imperial College of Science, Technology and Medicine and St Mary's Hospital, \\ London, England
}

D ifferential strain has been proposed to be a causative factor in failure of the supraspinatus tendon. We quantified the strains on the joint and bursal sides of the supraspinatus tendon with increasing load $\left(20\right.$ to $200 \mathrm{~N}$ ) and during $120^{\circ}$ of glenohumeral abduction with a constant tensile load ( 20 to $100 \mathrm{~N}$ ).

We tested ten fresh frozen cadaver shoulders on a purpose-built rig. Differential variable reluctance extensometers allowed calculation of the strain.

Static loading to $100 \mathrm{~N}$ or more increased strains on the joint side significantly more than on the bursal side. During glenohumeral abduction an increasing and significant difference in strain was measured between the joint and bursal sides of the supraspinatus tendon, which reached a maximum of $10.6 \%$ at abduction of $120^{\circ}$. The joint side strain of $7.5 \%$ reached values which were previously reported to cause failure.

Differential strain causes shearing between the layers of the supraspinatus tendon, which may contribute to the propagation of intratendinous defects that are initiated by high joint side strains.

J Bone Joint Surg [Br] 2003;85-B:594-9.

Received 16 January 2001; Accepted after revision 6 September 2002

In his 1998 Codman Lecture Fukuda ${ }^{1}$ drew particular attention to partial-thickness tears of the rotator cuff "because these conditions and their characteristics occupy a most sig-

P. Reilly, MS, FRCS, Clinical Research Fellow

A. A. Amis, DSc, FIMechE, Professor of Orthopaedic Biomechanics

Departments of Mechanical Engineering and Musculoskeletal Surgery

A. L. Wallace, PhD, FRACS, Senior Lecturer and Consultant in Orthopaedic Surgery

Department of Musculoskeletal Surgery

Imperial College London, South Kensington Campus, London SW7 2AZ, UK.

R. J. H. Emery, MS, FRCS, Consultant Orthopaedic Surgeon

Department of Orthopaedic Surgery, St Mary's Hospital, Praed Street, London W2 1NY, UK.

Correspondence should be sent to Professor A. A. Amis.

(C)2003 British Editorial Society of Bone and Joint Surgery doi:10.1302/0301-620X.85B4.12062 \$2.00 nificant position in the spectrum of rotator cuff disease". Many fundamental questions, however, relating to their pathogenesis, progression and symptomatology remain unanswered.

Cadaver $^{2}$ and radiological ${ }^{1}$ studies have suggested that intratendinous tears occur frequently, accounting for up to half of all partial-thickness tears. In 1939 Lindblom and Palmer $^{3}$ first considered the role of intratendinous shearing in the pathogenesis of tears of the rotator cuff. Since then a number of studies have suggested that the supraspinatus tendon has a heterogeneous structure and non-linear response to loading. ${ }^{4-7}$ The anterior third of the supraspinatus tendon was found to be thicker, with a higher ultimate load and failure stress than the posterior third. It was concluded that the anterior area was the strongest part and performed the main functional role of the tendon. ${ }^{6}$ It has also been shown that the joint and bursal layers of the tendon have different properties, with the joint side having a higher modulus of elasticity and lower ultimate strain. ${ }^{4}$

From engineering practice, it is clear that defects in a material can lead to concentrations of stress which may cause the defects to propagate. We hypothesised that the different mechanical properties of the joint and bursal regions of the supraspinatus tendon would lead to different tissue strains under load. If correct, shear stress concentration between the layers could lead to propagation of partialthickness tears of the supraspinatus tendon.

To our knowledge this is the first attempt to quantify the strains which occur in the critical zone of the supraspinatus tendon under different loading conditions in vitro.

\section{Materials and Methods}

Thirteen cadaver specimens were harvested and frozen within 24 hours of death. The mean age of the specimens was 69 years (48 to 80) and seven were from men and six from women. There was no premorbid history available. Each specimen consisted of the lateral $7 \mathrm{~cm}$ of the scapula and the proximal $15 \mathrm{~cm}$ of the humerus, along with the surrounding soft tissues after removal of the deltoid muscle.

The specimens were defrosted individually over 24 hours in normal saline-soaked bags. Throughout testing they were moistened with physiological saline. The rotator interval was opened, the long head of biceps was divided in the 

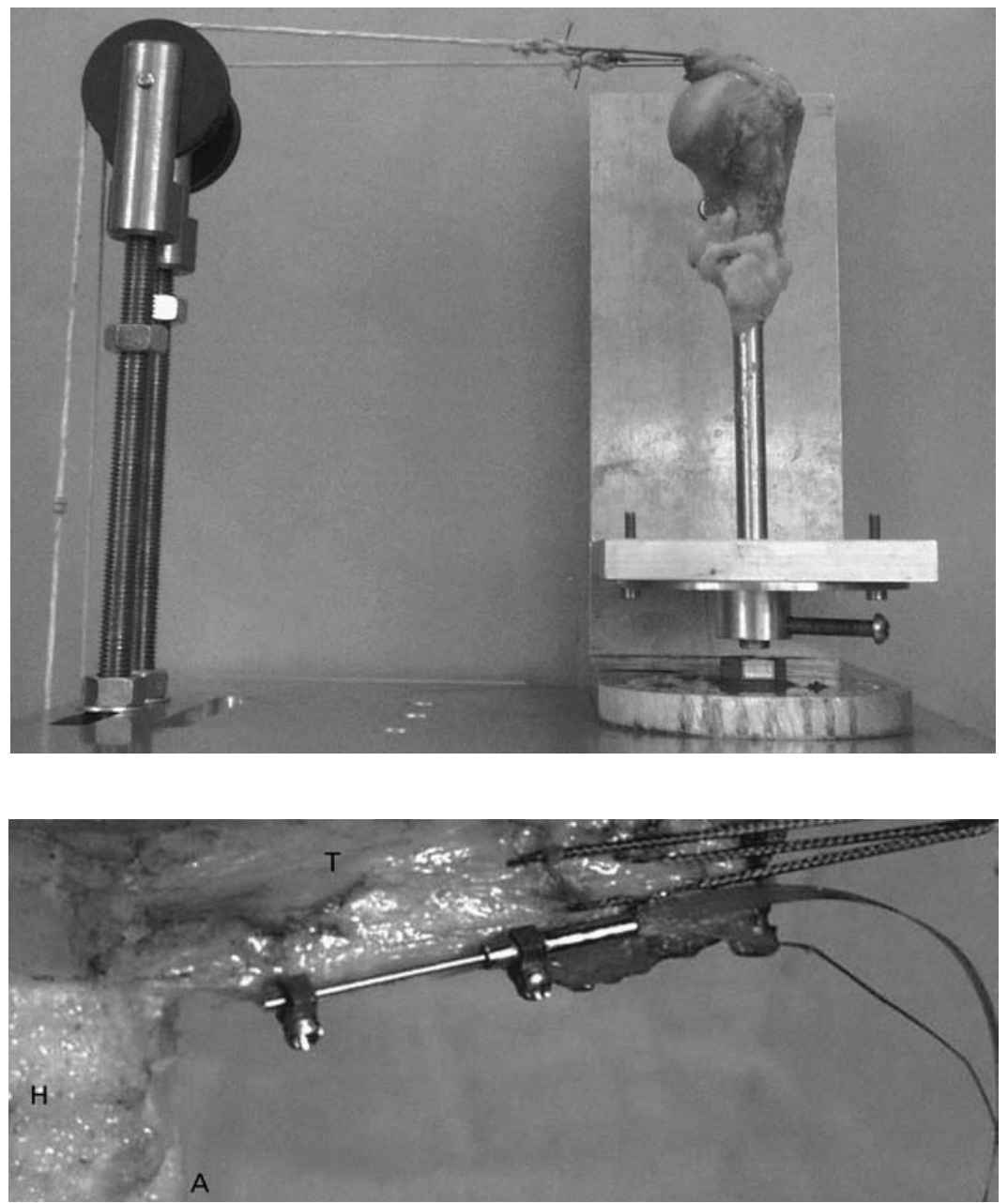

Fig. 1

Photograph of a specimen mounted on testing rig at $0^{\circ}$ of abduction. bicipital groove and subscapularis detached from the lesser tuberosity. The anterior border of supraspinatus was then accessible for instrumentation. The musculotendinous junction of infraspinatus was identified and the infraspinatus tendon was divided along its superior border. Infraspinatus and teres minor were then removed leaving the isolated supraspinatus tendon. At this stage three specimens were excluded from the study, in two because of macroscopic tears of the rotator cuff and in one a fracture of the humeral head.

A stainless-steel intramedullary rod $10 \mathrm{~mm}$ in diameter was fixed into the shaft of the humerus using polymethylmethacrylate bone cement. This allowed the specimen to be mounted on the purpose-built rig (Fig. 1). The testing apparatus was modified from a design used by Itoi et al. ${ }^{6}$ On this test rig the humerus could be rotated about three axes, horizontal, long and vertical. The horizontal and vertical axes allowed it to be rotated in abduction-adduction in any plane about the vertical axis. The specimen was mounted so that the centre of the head of the humerus was at the point of intersection of the three axes of rotation of the test rig. Rotational orientation of the specimen was standardised using the bicipital groove as a reference point.
Tensile load was applied to the supraspinatus tendon through a system of weights and pulleys. In order to ensure even distribution of load four mattress sutures (2 Ethibond; Ethicon Ltd, Edinburgh, UK) were placed in the musculotendinous junction. These were attached to weights by nylon cord.

Tendon strain was measured using linear differential reluctance transducers (DVRTs; Microstrain Inc, Burlington, Vermont). These were linear assemblies of a wire core sliding coaxially into the $8 \mathrm{~mm}$ long body of the DVRT. The core and the body were attached to the tissue through $3 \mathrm{~mm}$ long barbed pins. Any change in the length of the tissue caused the core to slide in the body, resulting in a change of output voltage. The DVRTs were placed in the joint and bursal portions of the anterior edge of the supraspinatus tendon, spanning the critical zone of Codman ${ }^{8}$ (Fig. 2). They were orientated parallel to the line of muscle action and were inserted with the humerus in neutral rotation and with $0^{\circ}$ of abduction. The specimen was pretensioned with a 20 $\mathrm{N}$ load which pulled the tendon medially before insertion of the DVRTs. The distance between the two barbs on insertion was the reference 'zero' position used in the calculation of tendon strain. 


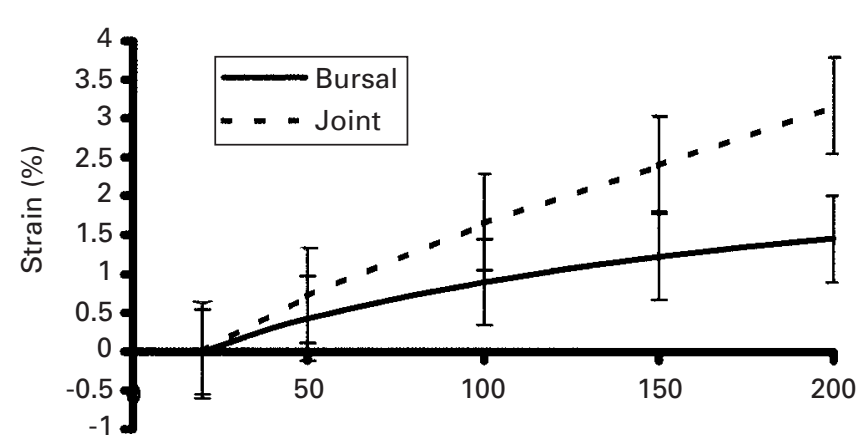

Load $(\mathrm{N})$

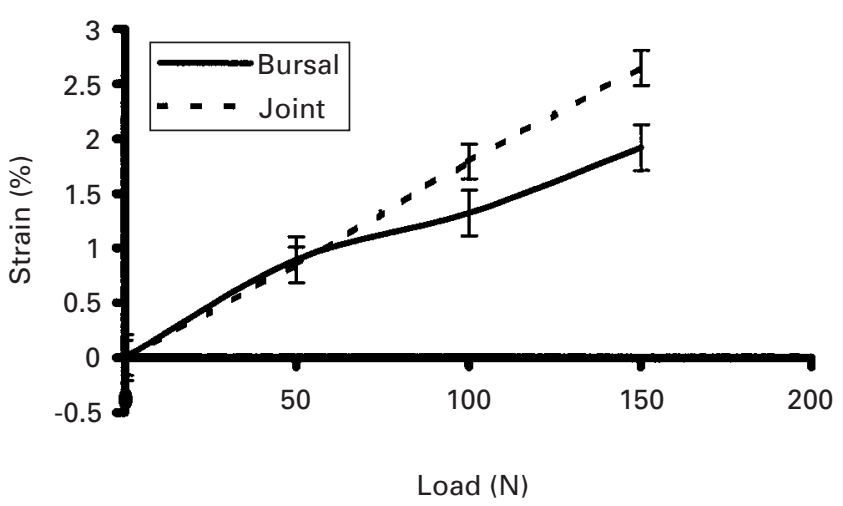

Fig. $3 b$

The mean supraspinatus tendon strains in the joint and bursal layers during tensile loading from 20 to $200 \mathrm{~N}$ at $0^{\circ}$ of abduction for both anterior (a) and posterior $(b)$ edges of the tendon. SEM bars are shown $(n=8)$. There is a significant difference $(p=0.01)$ at loads of $100 \mathrm{~N}$ and greater.

Troughs were made in the head of the humerus to prevent impingement of the DVRTs against the articular surface when the humerus was adducted. Each trough was approximately $20 \mathrm{~mm}$ long, $5 \mathrm{~mm}$ wide and $5 \mathrm{~mm}$ deep. They were positioned alongside the anterior and posterior borders of the supraspinatus tendon. The troughs did not extend under the tendon and thus its movement during the abduction and adduction of the humerus was unaltered.

Two sets of conditions were studied, namely, the application of an increasing load in a fixed position and abduction with a constant load. Pilot results had not shown significant hysteresis during testing cycles, but the results were always recorded with increasing load or abduction.

In order to minimise interspecimen variability, zero abduction with neutral rotation was chosen as the fixed position for the application of increasing tensile load. The loads used were $20,50,100,150$ and $200 \mathrm{~N}$. The system was preconditioned over three loading-unloading cycles, with output of the DVRTs recorded over ten further cycles.

The abduction testing used two tensile loads, $20 \mathrm{~N}$ to simulate passive movement in a subject with muscle tone, and $100 \mathrm{~N}$. The latter was chosen for two reasons. First, the tests at zero abduction showed a significant difference in strain at this level and, secondly, we wished to simulate active movement at a submaximal force of contraction of supraspinatus. ${ }^{9,10}$ The movement simulated was purely glenohumeral, $120^{\circ}$ representing full abduction when scapulothoracic movement was accounted for. The system was preconditioned by three cycles of full abduction-adduction before collection of data. During the ten cycles of testing, the voltage outputs of the DVRTs were recorded with the humerus stationary in increments of $30^{\circ}(0,30,60,90$ and $120^{\circ}$ ).

The DVRTs were then resited to similar positions on the posterior aspect of the tendon. The protocol was repeated with the exception of abduction with a load of $20 \mathrm{~N}$. The changes in voltage output from the DVRTs was converted to linear displacement using the calibration data supplied by the manufacturer (accurate to $\pm 10 \mu \mathrm{m}$ ).

The output of the DVRTs on insertion, i.e. the barb separation imposed by the insertion tool, was used to derive the initial length. This formed the basis of the calculation of strain as follows:

$$
\text { Strain }=\frac{\text { Change in length }}{\text { Initial length }}
$$

The ten strain values generated by each specimen for each position were used to calculate the mean bursal and joint side strains for each set of conditions. Complete sets of strain data were obtained for eight specimens.

Statistical analysis. Student's paired $t$-test was used for statistical analysis of differences between the joint and bursal layers because the positions of the DVRTs remained constant and the only change in the experimental conditions was either the load or the angle of abduction. Significance was achieved when $\mathrm{p}<0.0125$, with the Bonferroni correction factor for four tests in each experiment. In addition, the mean strain differences and the $99 \%$ confidence intervals (CI) for the strain differences were calculated at the data points at which significant differences were found.

\section{Results}

Increasing load at $0^{\circ}$ of abduction. Supraspinatus tendon strain increased with increasing static loading, as expected. This increase in strain was greater on the joint side of the tendon than on the bursal side. A statistically significant mean difference of $0.62 \%$ (99\% CI 0.19 to 1.05 ) between the strains in the joint and bursal layers was found in the anterior edge of the tendon at $100 \mathrm{~N}(\mathrm{p}=0.01)$ and above (Fig. 3). A similar trend was found at the posterior edge ( $\mathrm{p}=$ 0.03 at $100 \mathrm{~N})$ becoming significant by a load of $150 \mathrm{~N}(\mathrm{p}=$ $0.0088)$ with a mean strain difference of $0.73 \%(99 \% \mathrm{CI}$ 0.30 to 1.15 ).

When the strains in the anterior and posterior edges of the supraspinatus tendon were compared, it was found that 


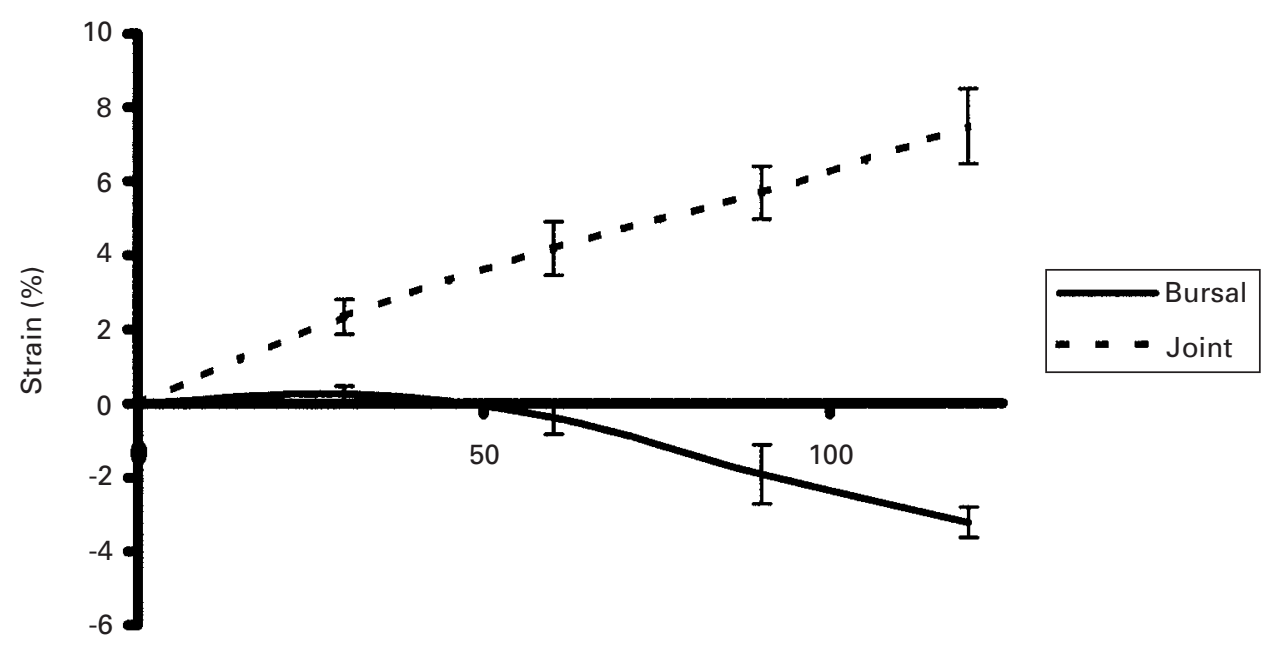

Abduction (degrees)

Fig. 4a

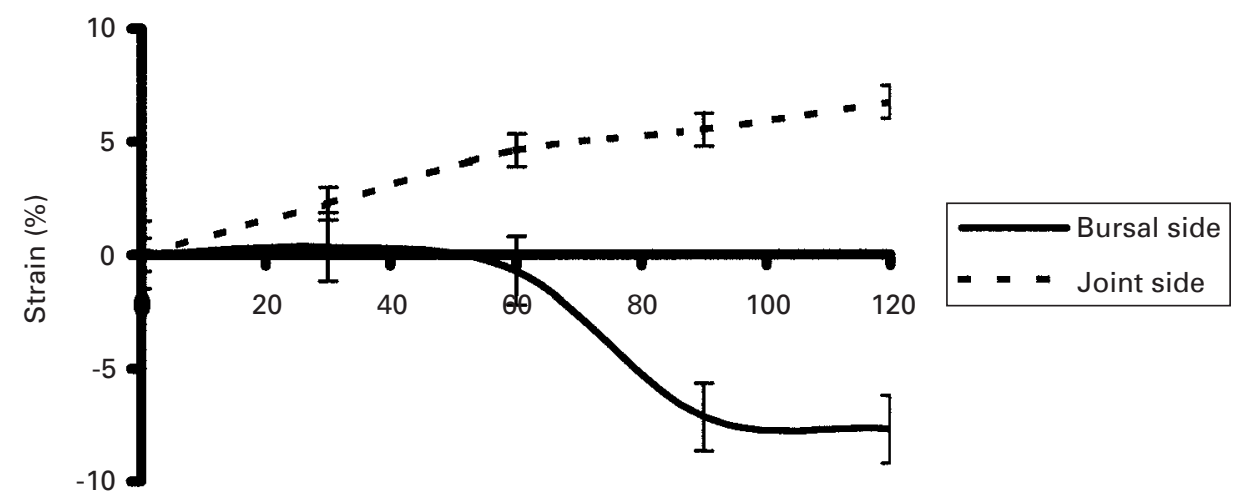

Abduction (degrees)

Fig. 4b

Anterior (a) and posterior (b) strains of the supraspinatus tendon in the joint and bursal layers during abduction from $0^{\circ}$ to $120^{\circ}$ with a load of $100 \mathrm{~N}$. There is a significant difference at abduction of $30^{\circ}$ and greater $(\mathrm{p} \leq 0.001)$. SEM shown $(\mathrm{n}=8)$.

the differences were not statistically significant in either layer of the tendon $(\mathrm{p}=0.50$ at $100 \mathrm{~N}$ on the bursal side; $\mathrm{p}=$ 0.31 at $100 \mathrm{~N}$ on the joint side).

Variation of strain with glenohumeral abduction. During abduction to $120^{\circ}$ the strain on the joint side increased and that on the bursal side decreased at both the anterior and posterior edges of the tendon. The difference between the layers was statistically significant at $30^{\circ}$ of abduction in the anterior edge of the tendon at both 20 and $100 \mathrm{~N}$ loads ( $\mathrm{p}<0.001$ ). With $20 \mathrm{~N}$ tension at $30^{\circ}$ of abduction, the mean strain difference was $2.16 \%(99 \% \mathrm{CI}$ 1.63 to 2.69 ). The difference in strain between the layers was also statistically significant at $30^{\circ}$ of abduction at the posterior edge $(\mathrm{p}<0.001$ at $100 \mathrm{~N})$, at which the mean strain difference was $1.92 \%$ (99\% CI 1.42 to 2.42 ). These differences increased with further abduction (Fig. 4). The joint side strain reached a mean of $7.5 \%$ at abduction of $120^{\circ}$.

We did not find a significant difference in strain from anterior to posterior during abduction ( $p=0.73$ at $90^{\circ}$ of abduction with $100 \mathrm{~N}$ load) in the joint side layer.

\section{Discussion}

Since Codman's seminal work $^{8}$ theories regarding the pathogenesis of tears of the rotator cuff have been much debated. He believed that the primary pathology was intrinsic, with avascularity in the "critical zone" of the supraspinatus leading to localised failure of the tendon which propagated to become a tear of the rotator cuff.

In 1939 Lindblom and Palmer ${ }^{3}$ first suggested that differential loading of the joint and bursal layers of the supra- 
spinatus tendon may result in a shearing effect between them and ultimately delamination of the tendon. Support for their hypothesis came later from Nakajima, ${ }^{4}$ who studied the material properties of the joint and bursal layers of the supraspinatus tendon in 40 cadaver specimens. He found that the modulus of elasticity of the bursal layer was significantly lower than that of the joint side layer $(p=0.05)$, whereas the strain to yield point and ultimate failing stress of the bursal layer were twice those of the joint side.

Although the concept of differential strain between the joint and bursal layers of the supraspinatus is not new, we are not aware of any previous measurements of strain which confirm it. The recent development of the miniature strain DVRTs provided the opportunity to test the hypothesis. In our study, increasing tendon tension at $0^{\circ}$ of humeral abduction resulted in a statistically significant difference in strain between the joint and bursal layers of the tendon, in agreement with the hypothesis of Lindblom and Palmer. ${ }^{3}$ The difference, however, in the resulting strains, was the reverse of what was expected. Since the modulus of elasticity of the joint side tendon layer has been found to be higher than that of the bursal, ${ }^{4}$ i.e. the material is stiffer, we expected to find lower strains on the joint side at the same load. The opposite result was found, with greater strains in the joint side layer. The combination of high strain measured in our experiment and the high stiffness reported by Nakajima ${ }^{4}$ suggests that most of the tension of the supraspinatus tendon was transmitted through the joint side, even when it might have been relatively relaxed, at $0^{\circ}$ of glenohumeral abduction. Although the material stiffness was not measured in our experiment, and so the joint layer of these specimens was not proven to be stiffer, the significantly higher strain still means that most of the load was carried by the joint side layer in these experiments.

We are not aware of previous attempts to measure strain directly in the bursal and joint side layers of the supraspinatus tendon during abduction of the humerus. The differential strains found indicate that intratendinous shearing forces are likely to increase with glenohumeral abduction. When maximally abducted, even with a relatively modest load of 100 $\mathrm{N}$, the mean joint side strain of $7.5 \%$ exceeded the previously published ${ }^{4}$ strain to yield point of $7 \%$. This is a potential explanation for the high incidence of partial-thickness tears on the joint side reported in athletes who are engaged in overhead throwing. ${ }^{11}$

The material properties of the different regions of the supraspinatus tendon have previously been analysed in the plane of the tendon. In the study of Itoi et $\mathrm{al}^{6}$ in which 11 fresh cadaver specimens were divided into three strips of equal width, a significantly higher modulus of elasticity was found in the anterior third of the tendon. They also found that the modulus of elasticity in the joint side layer exceeded that of the bursal side layer in the posterior third. We found no significant difference in strain between the anterior and posterior tendon, although we found the strains of the bursal side to be more variable than those of the joint side. The discrepancy in findings may be explained by differing experimental methodology. Itoi et $\mathrm{al}^{6}$ loaded the specimens to failure and calculated the modulus of elasticity between the musculotendinous junction and $2 \mathrm{~mm}$ proximal to the insertion. Our study used lower loads and concentrated on the 'critical zone' of the supraspinatus tendon, close to the bone attachment, based on the rationale that intratendinous tears are frequently found in this area. ${ }^{2}$

Our findings should be interpreted in the light of several limitations. First, we did not attempt to quantify the extent of pre-existing degenerative change in the tendons, although they were macroscopically normal. Since we used specimens from an elderly population, the extrapolation of results to a younger athletic population may not be justified. Secondly, we applied load to the cut end of the musculotendinous junction through mattress sutures, which were intended to spread the force evenly across its width. We do not know how well this replicated normal muscle action, but were not aware of any data which could suggest using a different method. Thirdly, we used the isolated supraspinatus tendon, thus removing some interactions with other structures, such as the rotator interval and the overlapping with infraspinatus. ${ }^{5}$ Finally, it was assumed that the line of action of the supraspinatus would remain in a constant relation to the scapula, and in the fixed plane of abduction of the test rig. Thus the external rotation of the humerus which accompanies terminal abduction in vivo was not modelled.

Nevertheless, the findings of our study have a direct relevance to tears of the supraspinatus tendon. We found that abduction of the humerus led to joint side strains at a level reported to cause failure of the tendon, ${ }^{4}$ despite tendon tension of only $100 \mathrm{~N}$. Therefore failure of the supraspinatus tendon from the joint side may be more likely to occur in full glenohumeral abduction. The difference in strain between the joint and bursal sides of the supraspinatus found with static loading and during abduction of the humerus implies a shearing effect between the two layers. This may cause small intratendinous defects to propagate into macroscopic delamination of the tendon with repeated physiological loading. Taken together, our results are consistent with the initiation of a tear on the joint side propagated in an interlaminar pattern ultimately resulting in a full-thickness tear.

Tears of the rotator cuff are the result of a number of biomechanical, biochemical and other physiological factors. The high joint side strain, combined with the statistically significant difference in strain between the joint and bursal layers of the supraspinatus tendon during abduction and with static loading, may account, at least in part, for the initiation and propagation of intratendinous tears of supraspinatus.

\footnotetext{
P. Reilly was a Research Fellow of The Royal College of Surgeons of Eng land. The Wishbone Trust and the Mason Medical Foundation gave financial support. Dr Itoi kindly helped with the design of the testing rig, and $\mathrm{Mr} \mathrm{K}$ Pickett built the testing rig. Dr Louise Brown, of the Clinical Trials Centre, Imperial College Faculty of Medicine, Charing Cross Hospital, provided statistical advice.

No benefits in any form have been received or will be received from a
} commercial party related directly or indirectly to the subject of this article. 


\section{References}

1. Fukuda H. Partial-thickness rotator cuff tears: a modern view on Codman's classic. J Shoulder Elbow Surg 2000;9:163-8.

2. Yamanaka K, Fukuda H, Hamada K, Nakajima T. Histology of the supraspinatus tendon with reference to rotator cuff tears. In: Gazielly D, Gleyze P, Thomas T, eds. The cuff, 15-18.

3. Lindblom K, Palmer I. Ruptures of the tendon aponeurosis of the shoulder joint. Acta Chir Scand 1939;82:133-42.

4. Nakajima T. Histological and biomechanical characteristics of the supraspinatus tendon. J Shoulder Elbow Surg 1994;3:79-87.

5. Clark JM, Harryman DT. Tendons, ligaments, and capsule of the rotator cuff: gross and microscopic anatomy. J Bone Joint Surg [Am] 1992;74-A:713-25.
6. Itoi E, Berglund LJ, Grabowski JJ, et al. Tensile properties of the supraspinatus tendon. J Orthop Res 1995;13:578-84.

7. Lee SB, Nakajima T, Luo ZP, et al. The bursal and articular sides of the supraspinatus tendon have a different compressive stiffness. Clin Biomech Bristol 2000;15:241-7.

8. Codman EA. The shoulder. 1934. Boston, Thomas Todd.

9. Wallace WA. Evaluation of the forces, the ICR and the neutral point during abduction and the shoulder. Trans ORS 1984.

10. Townsend H, Jobe FW, Pink M, Perry J. Electromyographic analysis of the glenohumeral muscles during a baseball rehabilitation program. Am J Sports Med 1991;19:264-72.

11. Altchek DW, Dines DM. Shoulder injuries in the throwing athlete. JAm Acad Orthop Surg 1995;3:159-65. 